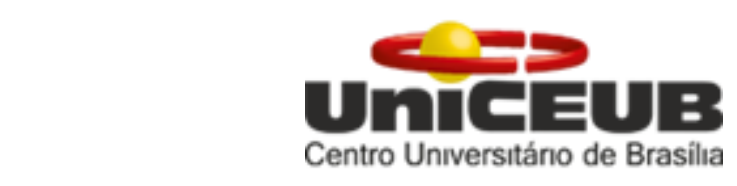

CENTRO UNIVERSITÁRIO DE BRASÍLIA - UniCEUB

PROGRAMA DE INICIAÇÃO CIENTÍFICA

KAROLINE LAURENTINO LOPES PINTO

RONAN WILK GUIMARÃES

ANÁLISE DE DISFUNÇõES TIREOIDIANAS INDUZIDAS PELO TRATAMENTO ONCOLÓGICO IMUNOTERÁPICO

BRASÍLIA 


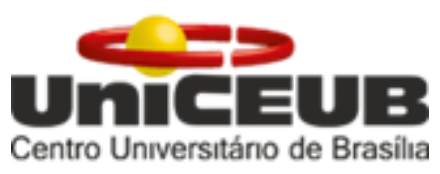

KAROLINE LAURENTINO LOPES PINTO

RONAN WILK GUIMARÃES

\section{ANÁLISE DE DISFUNÇÕES TIREOIDIANAS INDUZIDAS PELO TRATAMENTO ONCOLÓGICO IMUNOTERÁPICO}

Relatório final de pesquisa de Iniciação Científica apresentado à Assessoria de Pós-Graduação e Pesquisa.

Orientação: Profa. Dra. Gabriela Resende Vieira de Sousa

BRASÍLIA 


\section{RESUMO}

Introdução: Os inibidores das proteínas de checkpoint (IC), sobretudo os anti-PD-1, antiPD-L1 e anti-CTLA-4, têm sido cada vez mais utilizados na terapia contra o câncer. Em comparação com a terapêutica quimioterápica convencional, os ICs têm um perfil de toxicidade diferente, especialmente a ocorrência de efeitos adversos imunomediados (EAim) contra múltiplos sistemas, incluindo glândulas endócrinas. A compreensão dos eventos adversos relacionados ao tratamento dessas drogas é fundamental para a prática clínica e se detectados de modo precoce, podem ser reversíveis. Objetivos: O objetivo deste estudo foi descrever a incidência das principais disfunções tireoidianas secundárias ao tratamento com IC em um grupo de pacientes oncológicos em vigência de tratamento imunoterápico para diversos tipos de cânceres. Métodos: Estudo retrospectivo de pacientes diagnosticados com diferentes tipos de cânceres, submetidos a terapêutica com IC, entre fevereiro de 2015 a fevereiro de 2019, acompanhados no Centro de Oncologia Clínica do Hospital Sírio Libanês, unidade Brasília. Avaliou-se a função tireoidiana destes pacientes, através de dosagens hormonais de TSH e T4 livre ao longo do tratamento, a fim de verificar o desenvolvimento de disfunção tireoidiana. Resultados: Um total de 45 pacientes foram analisados, sendo excluídos 19. Dentre os demais pacientes, a prevalência de alteração nos níveis de TSH ou T4L ao longo do tratamento com Nivolumabe, foi de 7 pacientes $(58,33 \%)$, enquanto, apenas $5(41,66 \%)$ usuários de Pembrolizumabe apresentaram alterações nos níveis dos hormônios tireoidianos, sendo o hipotireoidismo a alterações mais prevalente no estudo. Além disso, não houve associação entre o tipo tumoral e a incidência de disfunções tireoidianas induzidas pela IC. Conclusão: As reações adversas envolvem qualquer órgão/tecido, sendo a disfunção tireoidiana umas das endocrinopatias mais frequentes. A monitorização da função tireoidiana através das dosagens hormonais favorece o diagnóstico e o tratamento precoce, com possível reversão dos sintomas e maior tolerância do paciente ao tratamento. Na população avaliada, encontrou-se uma incidência elevada de disfunções hormonais tireoidianas neste tipo de tratamento. Apesar dos resultados obtidos, novas pesquisas são necessárias para melhor entendimento sobre o desenvolvimento de doenças endócrinas com terapias inibidoras das proteínas de checkpoint.

Palavras-chaves: Imunoterapia. Efeitos colaterais e reações adversas relacionados a medicamentos. Doenças da glândula tireóide. 


\begin{abstract}
Background: Checkpoint proteins inhibitors (CI), especially anti-PD-1, anti-PD-L1 and anti-CTLA-4, have been used more often in cancer therapy. ICIs have a different toxicity profile in comparison to conventional chemotherapy, especially in relation to immunemediated adverse effects (EAim) against multiple systems, including endocrine glands. The understanding of adverse events related to the treatment of these drugs is fundamental to clinical practice and if detected early, they may be reversible. Objectives: the goal of this study is to describe the incidence of major thyroid dysfunctions that arise from the CI treatment, in subjects that are currently under immunotherapy treatment for different types of cancer. Methods: retrospective study of patients with different types of cancer submitted to CI therapy between 2015 February and 2019 February, at the Oncology Clinical Center of the Sírio Libanês Hospital, Unit of Brasília. Analyzing the thyroideal function through TSH and T4L dosation of those patients, before and after the treatment, verifying the development of thyroideal dysfunctionality. Results: A total of 45 patients were analyzed and 19 were excluded. The prevalence of alterations in TSH or T4L levels during treatment with Nivolumabe was 7 (58.33\%), whereas only 5 (41.66\%) users of Pembrolizumab presented alterations in thyroid hormone levels, hypothyroidism being the most prevalent one. There was no association between the cancer type and the incidence of thyroid dysfunction induced by CI. Conclusion: adverse reactions affect any tissue/organ, the thyroideal dysfunction is the most recurrent endocrinopathy. Monitoring the thyroid function through hormonal dosages favors early diagnosis and treatment, with possible reversion of symptoms and greater patient tolerance to treatment. In the evaluated population, a high incidence of thyroid hormonal dysfunctions was found. New research approaches are yet required for better understanding the development of endocrinopathies secondary to CI therapy.
\end{abstract}

Keywords: Immunotherapy. Drug-related side effects and adverse reactions. Thyroid diseases. 


\section{SUMÁRIO}

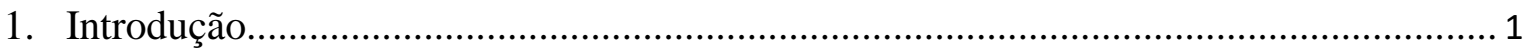

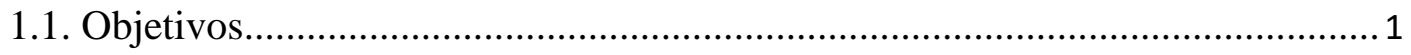

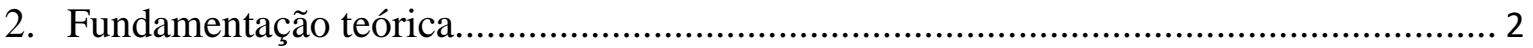

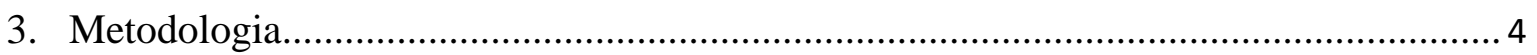

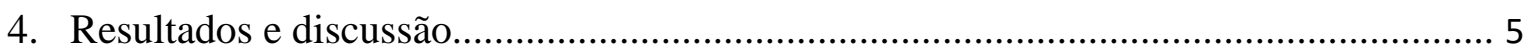

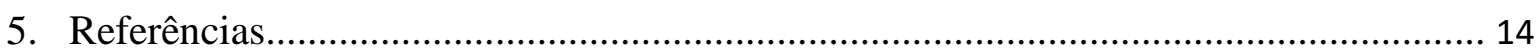

6. Anexo A - Aprovação do Comitê de Ética em Pesquisa................................................ 16 


\section{INTRODUÇÃO}

A evolução no entendimento sobre o funcionamento do sistema imune humano e o desenvolvimento de técnicas de sua modulação, a fim de que o próprio organismo combata o câncer, acarretaram o surgimento de uma nova era no tratamento oncológico (FRIEDMAN, 2016). Sendo assim, o uso de imunoterápicos, como o Nivolumabe, o Pembrolizumabe e o Ipilimumabe, cresceu e tem permitido o aumento na sobrevida global aos pacientes submetidos a essa terapêutica (LARKIN, 2015; TORINO, 2016).

Este estudo visou proporcionar melhor conhecimento das disfunções tireoidianas em doentes oncológicos tratados com inibidores das proteínas de checkpoints (IC), medicações em uso crescente para tratamento oncológico. Além disso, como os sintomas das disfunções tireoidianas, muitas vezes, são inespecíficos e podem ser semelhantes a sintomas das reações adversas comuns do tratamento oncológico, esse diagnóstico pode passar despercebido. É preciso melhor conhecimento sobre essa possibilidade para suspeição clínica, diagnóstico e tratamento das disfunções tireoidianas para o paciente em imunoterapia. Dessa forma, essas complicações são reconhecidas e tratadas precocemente, havendo consequente reversão dos sintomas e maior tolerabilidade do paciente ao tratamento (LARKIN, 2015).

\subsection{OBJETIVOS}

\section{GERAL}

Avaliar a incidência e as características clínicas do desenvolvimento de efeitos adversos imunes, principalmente tireoidopatias, em pacientes em tratamento imunoterápico de diferentes tipos de cânceres com Nivolumabe, Pembrolizumabe e Ipilimumabe.

\section{ESPECÍFICOS}

- Avaliar a incidência e prevalência de desenvolvimento de disfunções tireoidianas em pacientes com diagnóstico de câncer e tratamento imunoterápico com Nivolumabe, Pembrolizumabe e Ipilimumabe.

- Avaliar o perfil epidemiológico e o desenvolvimento de disfunções tireoidianas em pacientes com diagnóstico de câncer e tratamento imunoterápico com Nivolumabe, Pembrolizumabe e Ipilimumabe. 
- Avaliar o modo de evolução das disfunções tireoidianas induzidas pelo Nivolumabe, Pembrolizumabe e Ipilimumabe (definitivas ou transitórias).

\section{FUNDAMENTAÇÃO TEÓRICA}

Os inibidores das proteínas de checkpoints (IC), tais como o Nivolumabe, Pembrolizumabe e Ipilimumabe, fazem parte do atual arsenal de combate ao câncer. Ao invés de atacarem diretamente as células neoplásicas, esse tipo de tratamento tem como princípio atuar inibindo a apoptose dos linfócitos T (LT), possibilitando a proliferação e infiltração linfocitária nos tumores e potencializando assim o próprio organismo ao combate e posterior degradação das células cancerígenas. Desta forma, a imunoterapia tem se tornado uma opção terapêutica frequente contra cânceres agressivos como o melanoma metastático, o câncer de pulmão de células não pequenas e as recidivas de linfoma de Hodgkin (BRAHMER, 2018; RYDER 2014; SOUZA, 2010; TANAKA, 2016; TORINO, 2016).

As células das neoplasias malignas apresentam diversos mecanismos de evasão do sistema imunológico. Uma dessas formas é através da desregulação das proteínas de checkpoints, compostos participantes da resposta imune, que atuam como um minucioso mecanismo de regulação imune contra esse tipo de célula. Dessa maneira, a célula tumoral adquire capacidade de escape da apoptose ao desativar a célula $\mathrm{T}$, permitindo assim o crescimento e o desenvolvimento da neoplasia. A esse processo chama-se de resistência adaptativa dos tumores ao sistema imune. Entretanto, ao suprimir as proteínas de checkpoints, uma atividade antitumoral eficaz é gerada, combatendo a resistência adaptativa. A forma de atuação dos IC mais importantes trata-se da via do PD1/PD-L1, assim como, da via do CTLA-4, que podem ser moduladas com consequente aumento da resposta imune antitumoral. Os IC aprovados recentemente no Brasil para uso clínico, incluem o agente antiCTLA-4 (Ipilimumabe) e os agentes anti-PD-1 (Nivolumabe e Pembrolizumabe) (ALVES,2016; BRAHMER, 2018; TORINO, 2016).

Atualmente, existem vários ICs aprovados pela Food and Drug Administration dos EUA (FDA). O Ipilimumabe, um anticorpo anti-CTLA-4, foi o primeiro agente consentido para tratamento oncológico contra casos de melanoma avançado. Por sua vez, Pembrolizumabe e Nivolumabe, agentes anti-PD-1/PDL-1 foram aprovados para melanoma, câncer de pulmão não-pequenas células metastáticas, cânceres de cabeça e pescoço, 
carcinoma urotelial, adenocarcinoma gástrico, e tumores sólidos com deficiência de reparo incompatível, bem como para Linfoma de Hodgkin. O Nivolumabe, como monoterapia, está aprovado para utilização no carcinoma hepatocelular e em doentes com carcinoma das células renais. A combinação de Ipilimumabe e Nivolumabe para pacientes com melanoma avançado também recebeu aprovação da FDA (BRAHMER, 2018; HODI, 2010).

A imunoterapia, no entanto, pode desencadear o aparecimento de toxicidades inflamatórias e efeitos adversos imunomediados (EAim), consequência a inibição das proteínas de checkpoints e a proliferação de linfócitos T (CUKIER, 2017). Esses EAim são geralmente reversíveis, mas podem ser fatais em alguns casos se não detectados de modo precoce e não forem adequadamente tratados (BYUN, 2017; TRAINER, 2016). Na literatura, os sistemas e/ou órgãos com maior acometimento são o sistema gastrointestinal (colite), a pele (dermatite), o fígado (hepatite), o pulmão (pneumonite) e o sistema endócrino (tireoidite, hipofisite, adrenalite) (FRIEDMAN, 2016; MORGANSTEIN, 2017). Convém ressaltar ainda que os EAim podem ocorrer mesmo após a descontinuação do medicamento, necessitando de uma vigilância por um longo período após o término da medicação (TRAINER, 2016).

As reações adversas endócrinas são frequentes com o uso de imunoterápicos, correspondendo a cerca de 10 a 17\% dos casos (TRAINER, 2016). Segundo Iyer et al (2018), as alterações tireoidianas (hipotireoidismo, hipertireoidismo primário e tireoidite), induzidas tanto por anticorpos contra PD1, PDL-1 quanto por CTLA-4, tem uma prevalência variando de $6 \%$ a $20 \%$ de acordo com grandes ensaios clínicos de drogas em fase III de testes. A combinação de PD-1 e CTLA-4 desencadeia substancialmente mais EAim do que anti-PD-1 isoladamente (55\%-60\% vs 10\%-20\%) (WANG, 2018).

Tanto o hipotireoidismo, quanto o hipertireoidismo, assim como, a tireoidite (apresentação mais incomum), podem ocorrer com o uso de agentes anti-PD-1 em até 19\% dos casos, comparado com 9\% dos inibidores do CTLA-4. A Sociedade Brasileira de Oncologia Clínica (SBOC) recomenda realizar a avaliação da função hormonal tireoidiana antes de cada ciclo do medicamento ou mensalmente, e a cada 6-12 semanas por 6 meses após completar o tratamento. A terapêutica indicada, na vigência de alteração tireoidiana, é a reposição hormonal, no caso de hipotireoidismo, ou sintomáticos, diante a tireotoxicose, sendo, em algumas situações, necessário o tratamento imunossupressor (TRAINER, 2016). 


\section{METODOLOGIA}

A presente pesquisa foi um estudo observacional, transversal, analítico, retrospectivo, de fevereiro de 2015 a fevereiro de 2019. A amostra de participantes foi composta por maiores que 18 anos, de ambos os sexos, em acompanhamento no Centro de Oncologia Clínica do Hospital Sírio Libanês, unidade Brasília - DF, com diagnóstico de câncer como melanoma metastático, câncer de pulmão não pequenas células, linfoma de Hodgkin, câncer de cabeça e pescoço, entre outros, que iniciaram tratamento imunoterápico com Nivolumabe, Pembrolizumabe ou Ipilimumabe. Foram excluídos os pacientes que não aceitaram participar do estudo e que não tinham registro no prontuário das dosagens hormonais tireoidianas ao longo do tratamento. Os pacientes com diagnóstico prévio de disfunções autoimunes da tireoide foram excluídos da pesquisa. Porém, os mesmos foram analisados descritivamente devido este perfil de participantes ter relação com maior taxa de desregulação hormonal.

O objetivo deste estudo foi realizar uma análise retrospectiva das principais doenças tireoidianas secundárias ao tratamento imunoterápico em um grupo de pacientes oncológicos em vigência de tratamento imunoterápico, acompanhados no Centro de Oncologia Clínica do Hospital Sírio Libanês, unidade Brasília - DF. Além disso, foram avaliados os tipos de cânceres mais frequentes e comorbidades associadas, assim como, a evolução das disfunções tireoidianas induzidas pelo tratamento com Nivolumabe, Pembrolizumabe ou Ipilimumabe.

A participação do paciente foi apenas de consentir acesso da equipe pesquisadora aos seus dados do prontuário eletrônico. O Termo de Esclarecimento de Consentimento Livre Esclarecido (TCLE) foi aplicado aos pacientes que concordaram em participar, assim como, foram fornecidas orientações sobre a pesquisa aos mesmos. Foi realizada avaliação de dados como idade, sexo, comorbidades, tipo de câncer, presença atual ou história prévia de doenças autoimunes, medicamentos em uso que possam alterar a função tireoidiana.

Além disso, foram analisados resultados de exames laboratoriais como o hormônio estimulador da tireoide (TSH) e tiroxina livre (T4L), solicitados previamente ao início do tratamento do paciente com o imunoterápico, quando disponíveis. O paciente teve seus exames rotineiramente repetidos a cada ciclo do tratamento, anterior a administração do medicamento (Nivolumabe, Pembrolizumabe ou Ipilimumabe), com o objetivo de verificar o desenvolvimento de autoimunidade tireoidiana e/ou algum tipo de disfunção hormonal como hipotireoidismo, hipertireoidismo e tireoidite. 
Os valores de referência do hormônio tireoestimulante (TSH) e a tiroxina livre (T4L) foram definidos como 0,55 a 4,78 $\mathrm{mcUI} / \mathrm{ml}$ e 0,74 a $1,72 \mathrm{ng} / \mathrm{dl}$, respectivamente, realizados pelo método de quimioluminescência e o material soro usado pelo laboratório clínico Sabin, registrado no Conselho Regional de Farmacologia do Distrito Federal sob o número 03/000054.

Neste estudo considerou-se como diagnóstico de hipotireoidismo primário aqueles pacientes que apresentavam valores de TSH aumentado e de T4L reduzido de acordo com os valores de referência estabelecidos. O hipotireoidismo subclínico foi definido pela presença de níveis séricos elevados de TSH em face de concentrações séricas normais de T4L. Os casos que evoluíram com alterações tireoidianas e TSH abaixo do valor de referência foram considerados dentro de um grupo caracterizado por hipertireoidismo ou tireotoxicose, devido não ter sido possível determinar com exatidão a etiologia das alterações, visto que os paciente não tinham exames como TRAB e cintilografia da tireoide.

Foram realizadas análises descritivas para delinear a incidência e prevalência de alterações tireoidianas induzidas pelos imunoterápico.

O presente projeto encontra-se aprovado na Plataforma Brasil sobre o número de CAAE 74772317.5.0000.0023 pelo CEP/ UniCEUB e pelo CEP/Hospital Sírio Libanês, com o número de CAAE 74772317.5.3001.5461.

De acordo com os princípios éticos em pesquisa com seres humanos, os autores tomaram todas as precauções para que a confidencialidade, a privacidade e a autonomia dos sujeitos da pesquisa fossem preservadas. Os pacientes que aceitaram participar do estudo assinaram um TCLE, no qual os pesquisadores esclareceram quanto à importância do estudo, assim como, os benefícios e os riscos da pesquisa.

\section{RESULTADOS E DISCUSSÃO}

Foram selecionados 45 pacientes em tratamento com Nivolumabe, Pembrolizumabe ou Ipilimumabe, entre o período de fevereiro de 2015 até fevereiro de 2019, do Centro de Oncologia Clínica do Hospital Sírio Libanês, unidade Brasília - DF. Desta amostra, 19 pacientes foram excluídos da pesquisa, sendo que 10 destes, o motivo foi a ausência de disponibilidade dos exames de função tireoidiana durante o intervalo de tempo estabelecido do tratamento. Dois destes 10 pacientes foram excluídos devido ao óbito do paciente ocorrer próximo ao início do tratamento com o imunoterápico, impossibilitando a coleta dos exames 
tireoidianos. Além disso, outros 9 pacientes foram excluídos devido a distúrbio prévio da tireoide. Dos excluídos da pesquisa, 8 usavam Nivolumabe, 10 usavam Pembrolizumabe, 1 usava Ipilimumabe e 1 fez associação de Nivolumabe com Ipilimumabe. Sendo assim, foram coletados dados de 26 pacientes (Figura 1).

Figura 1 - Caracterização da seleção da amostra

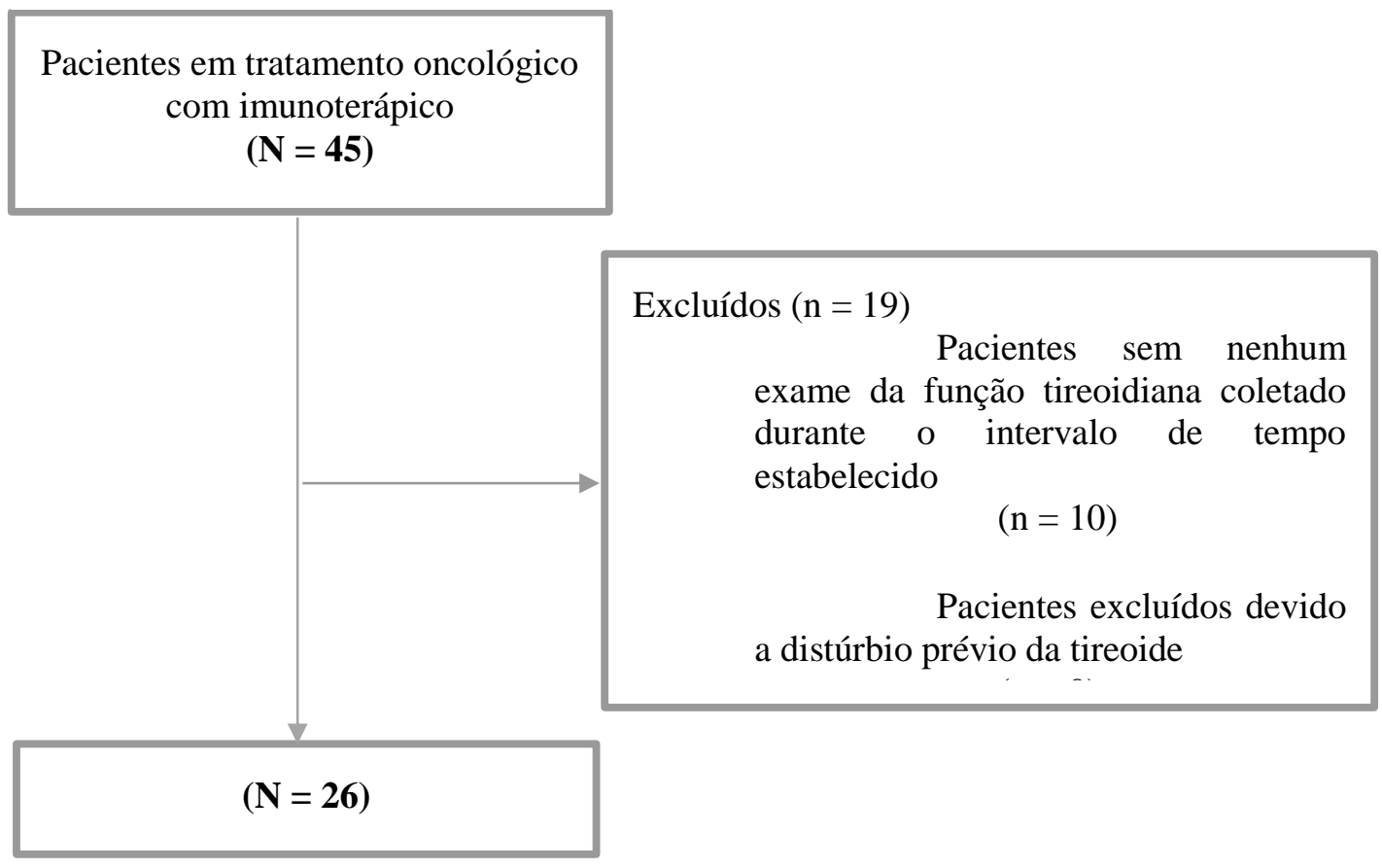

Fonte: Elaboração do autor baseado em prontuários do Hospital Sírio Libanês

A amostra foi separada em grupos de acordo com o uso específico do imunoterápico. O grupo que fez uso do Nivolumabe totalizou 20 esquemas terapêuticos, sendo que, 12 foram analisados após os critérios de exclusão, 7 do gênero masculino e 5 do feminino com idade média de 68,25 anos. Destes, apenas 3 (25\%) tinham exames hormonais prévios ao início do tratamento. A prevalência de alteração nos níveis de TSH ou T4L, ao longo do estudo neste grupo, foi 7 pacientes $(58,33 \%)$. Na análise dos exames hormonais tireoidianos com três meses após o início do uso do Nivolumabe, foi constatado $1(8,33 \%)$ caso de alteração tireoidiana, já na coleta após o terceiro mês até um ano do início do quimioterápico, 6 (50\%) dos pacientes apresentaram alteração no TSH. Dos participantes que fizeram alterações tireoidianas, a maioria $(41,66 \%)$ apresentou hipotireoidismo subclínico e 2 (16,66\%) hipertireoidismo ou tireotoxicose, evidenciado na Tabela 1. 
Tabela 1 - Alterações hormonais tireoidianas com o uso de Nivolumabe e Pembrolizumabe

\begin{tabular}{|c|c|c|}
\hline & Nivolumabe $(\mathrm{n}=12)$ & Pembrolizumabe (n=12) \\
\hline $\begin{array}{c}\text { Pacientes que apresentaram alterações } \\
\text { nos exames laboratoriais (TSH, T4L) }\end{array}$ & $7(58,33 \%)$ & $5(41,66 \%)$ \\
\hline Hipertireoidismo ou tireotoxicose & $2(16,66 \%)$ & --- \\
\hline Hipotireoidismo clínico & --- & $2(8,33 \%)$ \\
\hline Hipotireoidismo subclínico & $5(41,66 \%)$ & $2(16,66 \%)$ \\
\hline $\begin{array}{c}\text { Hipertireoidismo ou tireotoxicose } \\
\text { seguido de hipotireoidismo }\end{array}$ & --- & $4(33,33 \%)$ \\
\hline $\begin{array}{c}\text { Alterações dos exames nos primeiros } \\
\text { três meses de tratamento }\end{array}$ & $1(8,33 \%)$ & $1(8,33 \%)$ \\
\hline $\begin{array}{c}\text { Alterações dos exames após três meses } \\
\text { a um ano de tratamento }\end{array}$ & $6(50 \%)$ & $2(16 \%)$ \\
\hline
\end{tabular}

Fonte: Elaboração do autor baseado em prontuários do Hospital Sírio Libanês

Segundo Sousa et al (2018), a incidência de disfunção tireoidiana em pacientes tratados sob regime de monoterapia foi maior naqueles que fizeram uso de agentes anti-PD1. O mesmo afirma Ryder et al (2014), em que a ocorrência de alteração tireoidiana induzida pelos agentes anti-PD-1 é de apenas 3\%, taxa inferior à encontrada neste estudo (Tabela 1). No entanto, o número de indivíduos tratados com agentes anti-PD-1 nesta pesquisa é pequeno, sendo necessário outros estudos, com maior número de pacientes, para determinar a frequência e a gravidade dos EAims relacionados à tireoide induzidas pelo Nivolumabe e/ou Pembrolizumabe.

O tipo de câncer que levou à indicação do uso de Nivolumabe foi o adenocarcinoma de pulmão, correspondendo $7(58,33 \%)$ da amostra. Em segundo lugar, houve maior prevalência do câncer de cabeça e pescoço com 2 (16,66\%) dos casos, seguido pelo melanoma com $1(8,33 \%)$ caso, e outros tipos de cânceres como o carcinoma basocelular e o Linfoma de Hodgkin, com 2 (16,66\%) casos (Tabela 2). As comorbidades mais prevalentes nesse grupo foram doença pulmonar obstrutiva crônica (DPOC) com 6 casos, seguida da 
doença arterial com 3, e dislipidemia com 2, hipertensão arterial sistêmica (HAS), depressão e diabetes mellitus (DM) com 1 caso cada uma (Tabela 3).

Tabela 2 - Correlação entre os tipos de cânceres e o tratamento oncológico com Nivolumabe e Pembrolizumabe

\begin{tabular}{|c|c|c|}
\hline Tipos de câncer & Nivolumabe $(\mathrm{n}=12)$ & Pembrolizumabe (n = 12) \\
\hline Adenocarcinoma de pulmão & $7(58,33 \%)$ & $4(33,33 \%)$ \\
\hline Câncer de cabeça e pescoço & $2(16,66 \%)$ & $4(33,33 \%)$ \\
\hline Melanoma & $1(8,33 \%)$ & $4(33,33 \%)$ \\
\hline $\begin{array}{c}\text { Outros } \\
\text { carcinoma basocelular, câncer de } \\
\text { bexiga, carcinoma cutâneo, } \\
\text { carcinoma urotelial e Linfoma de } \\
\text { Hodgkin) }\end{array}$ & $2(16,66 \%)$ & \\
\hline
\end{tabular}

Fonte: Elaboração do autor baseado em prontuários do Hospital Sírio Libanês

Tabela 3 - Comorbidades nos pacientes em uso de Nivolumabe e Pembrolizumabe

\begin{tabular}{|c|c|c|}
\hline Comorbidades & Nivolumabe $(\mathrm{n}=12)$ & Pembrolizumabe $(\mathrm{n}=12)$ \\
\hline DPOC & $6(50 \%)$ & $5(41,66 \%)$ \\
\hline DAC & $3(25 \%)$ & $3(25 \%)$ \\
\hline Dislipidemia & $2(16,66 \%)$ & $1(8,33 \%)$ \\
\hline HAS & $1(8,33 \%)$ & $6(50 \%)$ \\
\hline DM & $1(8,33 \%)$ & $2(16,66 \%)$ \\
\hline Depressão & $1(8,33 \%)$ & $2(16,66 \%)$ \\
\hline
\end{tabular}

Fonte: Elaboração do autor baseado em prontuários do Hospital Sírio Libanês 
O grupo que fez uso do Pembrolizumabe totalizou 22 pacientes, sendo que, 12 foram analisados após os critérios de exclusão, 6 do gênero masculino e 6 do feminino com idade média de 73 anos. Destes, apenas $8(66,66 \%)$ tinham exames hormonais prévios ao início do tratamento. A prevalência de alteração nos níveis de TSH ou T4L, ao longo do estudo neste grupo, foi 5 pacientes $(41,66 \%)$. Na análise dos exames hormonais tireoidianos com três meses após o início do uso do Pembrolizumabe foi constatado 4 (33,33\%) casos de alteração tireoidiana, já na coleta após o terceiro mês até um ano do início do quimioterápico, apenas $1(8,33 \%)$ dos pacientes apresentaram alteração no TSH. Dentre os participantes que fizeram alterações tireoidianas, $2(16,66 \%)$ pacientes apresentaram hipertireoidismo ou tireotoxicose com evolução para hipotireoidismo na dosagem hormonal subsequente, 2 (16,66\%) hipotireoidismo subclínico e 1 (8,33\%) hipotireoidismo, evidenciado na Tabela 1.

Nesta casuística, as alterações mais comuns da função tireoidiana detectadas foram hipotireoidismo primário e hipotireoidismo subclínico. A maior incidência de hipotireoidismo primário foi constatada naqueles que receberam terapia com Pembrolizumabe, apresentando incidência de 8,33\%, dado que que se correlacionou com o que foi relatado em outros ensaios clínicos (PATEL, 2018). Segundo a literatura, a terapia com Pembrolizumabe tem sido associada com uma incidência de 57-79.5\% de eventos adversos imunomediados, entre os quais hipotireoidismo (5-10.1\%) e hipertireoidismo $(<2$ 6.5\%) (GARON, 2015; HERBST, 2016).

Os tipos de tumores que mais levaram à indicação do uso de Pembrolizumabe foram $4(33,33 \%)$ casos de adenocarcinoma de pulmão, 4 (33,33\%) casos de melanoma e outros tipos de cânceres como o carcinoma de bexiga, Linfoma de Hodgkin, carcinoma cutâneo e carcinoma urotelial, com $4(33,33 \%)$ casos (Tabela 2). As comorbidades mais prevalentes nesse grupo foram HAS, com 6 casos, seguida da DPOC, com 5, doença arterial crônica, com 3, DM com 2, depressão com 2 e dislipidemia com 1 caso (Tabela 3).

As disfunções tireoidianas, ocasionadas pelo agente anti-CTLA-4, apresentam uma prevalência de 3\%, conforme Weber et al (2012). Este dado, segundo o autor, mostra um padrão de ocorrência comum, sendo que as alterações endócrinas costumam surgir de modo tardio, em comparação a alterações na pele, no fígado e no sistema gastrointestinal, após início da terapêutica com o Ipilimumabe. Nesta casuística, a ocorrência de alterações na tireoide com Ipilimumabe como monoterapia foi de apenas um caso, demonstrando necessidade de novos estudos com maior número de pacientes, assim como, melhores relatos em prontuário das disfunções tireoidianas para melhor discussão dos achados clínicos. 
Dois pacientes analisados fizeram uso de terapêutica conjunta com Ipilimumabe e Nivolumabe, um homem e uma mulher, com idade média de 64 anos e diagnóstico de melanoma e adenocarcinoma de pulmão, respectivamente. Esses dois pacientes apresentaram alterações nos hormônios tireoidianos nos exames coletados após 3 meses do uso dos imunoterápicos, sendo o primeiro hipotireoidismo subclínico e o segundo hipertireoidismo ou tireotoxicose com evolução para hipotireoidismo.

De acordo com Sousa et al (2018), a incidência de EAim endócrinos com o uso de IC é significativamente maior em pacientes sob terapia combinada em comparação com pacientes tratados com monoterapia. Apesar da amostra deste estudo ser pequena, observouse, como constatado na literatura, que houve alterações da disfunção tireoidiana com uso de terapêutica combinada, apresentando alterações dos exames laboratoriais (TSH e T4L) após início do uso dos IC de modo precoce e significativo (Tabela 1).

Ryder et al (2014) afirma que, o uso concomitante de Nivolumabe e Ipilimumabe pode ocasionar aumento do número de casos de hipotireoidismo e/ou tireoidite, sendo a incidência de cerca de $22 \%$ dos casos de tireoidite e/ou hipotireoidismo. Dado que corrobora com o observado neste estudo, em que dois pacientes, que fizeram a terapêutica concomitante, apresentaram disfunção tireoidiana após início do tratamento imunoterápico, assim como, o participante excluído em decorrência de alteração tireoidiana prévia.

Destaca-se que o hipertireoidismo é uma alteração menos comumente relatada, dado observado neste estudo com prevalências baixas. Esta alteração deveu-se possivelmente por uma tireoidite indolor prévia. Esse quadro geralmente não requer o uso de fármacos antitireoidianos, podendo evoluir para hipotireoidismo e necessidade da monitorização dos níveis hormonais do paciente posteriormente (O'MALLEY, 2017; CUKIER, 2017; TORINO, 2016; ROSS, 2016; SPAIN, 2016). O mesmo fato foi detectado no estudo de Orlov et al (2015) no qual foi observado uma série de casos de 10 pacientes com tireoidite indolor, sob terapia com anti-PD-1, e todos foram diagnosticados com hipotireoidismo, sendo este quadro precedido por tirotoxicose transitória em seis dos pacientes.

A baixa prevalência de hipertireoidismo ou tireotoxicose constatada no estudo devese, provavelmente, a frequência irregular de solicitação dos exames de função tireoidiana. Consequentemente houve maior diagnóstico de hipotireoidismo que de hipertireoidismo ou tireotoxicose.

Apesar de relatos da literatura afirmarem que há maior prevalência de EAim associado ao uso de agentes anti-PD-1 nos primeiros 4 meses de tratamento (WEINSTEIN, 
2017), o presente estudo observou maiores alterações nos meses subsequentes, principalmente, entre 3 meses a 1 ano de tratamento com Nivolumabe e Pembrolizumabe.

Além disso, não houve associação entre o tipo tumoral e a incidência de disfunções tireoidianas induzidas pela IC, dado também constatado no estudo de metanálise de Sousa et al (2018). A média da idade dos pacientes em análise foi de 70,11 anos, o que coincide com a idade de maior incidência dos tumores em tratamento.

As comorbidades mais frequentes também não se correlacionaram com o uso de imunoterápicos, mas possuem em comum alguns fatores de risco para desenvolvimento dos tipos de cânceres mais frequentes em tratamento, como é o caso do DPOC, HAS e DAC.

Do total dos 26 pacientes analisados, 14 (53,84\%) apresentaram alterações laboratoriais do hormônio TSH ao longo do tratamento. Porém, por falta de relatos em prontuário, não foi possível saber se a terapêutica para hipotireoidismo, hipertireoidismo ou tireotoxicose foi realizada, a fim de reverter as complicações tireoidianas apresentadas pós uso dos IC, sendo esta uma das dificuldades encontradas nesta presente pesquisa.

Segundo a literatura, o surgimento de distúrbios da tireoide decorrentes da terapia com IC podem ser manejados sem a necessidade de suspender a medicação imunoterápica (2018). Os pacientes com hipotireoidismo primário podem começar a reposição hormonal tireoidiana com a dose $0,8 \mu \mathrm{g} / \mathrm{kg} / \mathrm{dia}$ de levotiroxina. Em caso de pacientes idosos e de pacientes com doenças cardíacas, é recomendado iniciar com doses mais baixas de

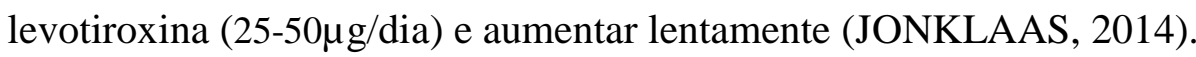

Já o manejo terapêutico da tireotoxicose advinda do uso de IC pode ser conduzido com o uso de $\beta$-bloqueadores por um breve período, conforme seja necessário para controlar os sintomas de ativação simpática. A indicação para iniciar o $\beta$-bloqueador é a identificação da frequência cardíaca acima de 100 batimentos por minuto e uma pressão arterial sistêmica que não seja baixa (JONKLAAS, 2014).

Além disso, como a maioria dos casos de hipertireoidismo ou tireotoxicose evolui para hipotireoidismo em uma média de 6 semanas, os níveis de TSH e T4L devem ser monitorados a cada 2-3 semanas (SOUSA, 2018). Para os idosos com risco cardiovascular basal elevado que apresentam tireotoxicose grave, um ciclo curto de alta dose de corticoide sistêmico ( $1 \mathrm{mg} / \mathrm{kg} /$ dia de prednisona oral) pode ser utilizado por até 14 dias (JONKLAAS, 2014).

Como forma de acompanhamento das alterações tireoidianas induzidas pelos ICs, o perfil dos hormônios TSH e T4L deve ser dosado antes de cada dose do imunoterápico ou 
mensalmente após o início do mesmo como forma de rastreamento dos EAim. Já a sua monitorização após a identificação de alterações tireoidianas não deve passar um mês após o início das medidas terapêuticas para posterior titulação e ajuste das doses (WEINSTEIN, 2017).

Ao analisar os 9 esquemas terapêuticos excluídos devido ao distúrbio prévio da tireoide, mas que se encontravam com os níveis hormonais do TSH e do T4L dentro da normalidade, mediante uso da medicação para a tireoide, observou-se que $5(55,55 \%)$ pacientes apresentaram alterações tireoidianas após início do uso dos imunoterápicos. Dentre os 4 usuários de Nivolumabe, 3 apresentaram desajuste nos níveis hormonais, compondo um caso com elevação de TSH e 2 casos com diminuição de TSH, provável tireoidite; e um deles evoluiu posteriormente para hipotireoidismo. Outros 3 pacientes estavam em uso de Pembrolizumabe e um deles apresentou hipotireoidismo subclínico com necessidade do ajuste da medicação usada anteriormente. Além disso, um paciente em uso de Ipilimumabe não apresentou alterações e outro paciente, que fez uso da associação de Nivolumabe com Ipilimumabe, apresentou hipotireoidismo.

Apesar da exclusão de 9 pacientes da amostra devido estes apresentarem distúrbio prévio da tireoide controlado, o estudo de coorte retrospectivo de Patel et al (PATEL, 2018), afirma que existe uma alta probabilidade das alterações tireoidianas basais tornarem-se exacerbadas nos pacientes em terapia com IC, fato também observado na presente pesquisa no qual foi constatado que $5(55,55 \%)$ pacientes apresentaram alterações tireoidianas após início do uso dos imunoterápicos.

Este estudo apresenta certas limitações. O uso da imunoterapia no tratamento oncológico é um tema recente e, na época do início da pesquisa, ainda não existia um protocolo ou Diretriz com indicação bem estabelecida quanto a solicitação dos exames tireoidianos de rotina e sua monitorização para todos os pacientes. Portanto, não fazia parte da prática de todos os médicos solicitá-los de forma rotineira durante o tratamento imunoterápico, o que inviabilizou a inclusão de diversos pacientes por falta de exames prévios ao início do tratamento com os ICs.

Além disso, o preenchimento incompleto dos prontuários pelos médicos dificultou a coleta de dados pelos pesquisadores. Por consequência, alguns pacientes tiveram que ser excluídos da pesquisa pela falta de dados, assim como, não foi possível averiguar se os pacientes que apresentaram alterações tireoidianas foram adequadamente tratados com medicações antitireoidianas. 
As terapias com uso de inibidores das proteínas de checkpoint, principalmente, Nivolumabe, Pembrolizumabe e Ipilimumabe, foram recentemente aprovadas por órgãos reguladores tanto internacionais (FDA) quanto nacionais (Anvisa) a fim de otimizar o tratamento oncológico. Portanto, espera-se um aumento significativo do uso destas medicações na prática clínica, assim como, relatos de casos de efeitos adversos imunomediados.

As reações adversas envolvem qualquer órgão/tecido, sendo a alteração cutânea a mais frequente, porém a disfunção tireoidiana é a endocrinopatia que ocorre de modo tardio com o uso da maioria dos imunoterápicos.

Esta análise ilustra que o hipotireoidismo é o efeito adverso mais prevalente após início do uso da imunoterapia sobretudo com Nivolumabe. Dado observado em decorrência do monitoramento necessário dos níveis séricos de TSH, T4 livre em paciente submetidos ao tratamento oncológico.

Além disso, não foi observada associação entre o tipo tumoral e a incidência de disfunções tireoidianas, assim como, as comorbidades foram constatadas como fatores de risco para os cânceres diagnosticados e utilizados como fonte de estudo.

O manejo ideal das EAim é baseado na experiência clínica, pois há poucos estudos prospectivos realizados para avaliar a melhor estratégia de tratamento diante a EAim. E apesar da presença desses efeitos, a terapêutica com imunoterápicos, frequentemente, não precisará ser suspensa.

Apesar dos resultados obtidos, novas pesquisas são necessárias para melhor entendimento de quais pacientes são mais suscetíveis ao desenvolvimento de doenças endócrinas com terapias inibidoras das proteínas de checkpoint utilizadas no tratamento contra o câncer. 


\section{REFERÊNCIAS}

ALVES, V. S. S.; GUEDES, V. ANTI-PD-1 E ANTI-PD-L1: Novas perspectivas para o tratamento de diversos tipos de câncer. Revista de Patologia do Tocantins, v. 3, n. 1, p. 2443, 2016.

BRAHMER, J. R. et al. Management of immune-related adverse events in patients treated with immune checkpoint inhibitor therapy: American Society of Clinical Oncology Clinical Practice Guideline. Journal of clinical oncology: official journal of the American Society of Clinical Oncology, Alexandria, v. 36, n. 17, p. 1714-1767, 2018.

BYUN, D. J. et al. Cancer immunotherapy - immune checkpoint blockade andassociated endocrinopathies. Nat Rev Endocrinol, London, v. 13, n. 4, p. 195-207, apr. 2017. doi: 10.1038/nrendo.2016.205.

CUKIER, P. et al. Endocrine side effects of cancer immunotherapy. Endocrine-related cancer, Grã-Bretanha, v. 24, n. 12, p. T331-T347, 2017.

FRIEDMAN, C. F.; PROVERBS-SINGH, T. A.; POSTOW, M. A. Treatment of the immune-related adverse effects of immune checkpoint inhibitors: a review. JAMA oncology, Washington, v. 2, n. 10, p. 1346-1353, 2016.

GARON, E. B. et al. Pembrolizumab for the treatment of non-small-cell lung cancer. N Engl J Med, Waltham, v. 372, n. 21, p. 2018-2028, 2015.

HERBST, R. S. et al. Pembrolizumab versus docetaxel for previously treated, PD-L1positive, advanced non-small-cell lung cancer (KEYNOTE-010): a randomised controlled trial. The Lancet, London, v. 387, n. 10027, p. 1540-1550, apr. 2016.

HODI, F. S. et al. Improved survival with ipilimumab in patients with metastatic melanoma. N Engl J Med, Waltham, v. 363, n. 8, p. 711-723, aug. 2010.

IYER, P. C. et al. Immune-related thyroiditis with immune checkpoint inhibitors. Thyroid, Falls Church, v. 28, n. 10, p. 1243-1251, 2018.

JONKLAAS J. et al. American Thyroid Association Task Force on Thyroid Hormone Replacement. Guidelines for the treatment of hypothyroidism: prepared by the American Thyroid Association Task Force on Thyroid Hormone Replacement. Thyroid, Falls Church, v. 24, n. 12, p. 1670-1751, 2014.

LARKIN, J. et al. Combined nivolumab and ipilimumab or monotherapy in untreated melanoma. N Engl J Med, Waltham, v. 373, n. 1, p. 23-34, jul. 2015.

MORGANSTEIN, D. L. et al. Thyroid abnormalities following the use of cytotoxic Tlymphocyte antigen-4 and programmed death receptor protein-1 inhibitors in the treatment of melanoma. Clin Endocrinol (Oxf), Hoboken, v. 86, n. 4, p. 614-620, apr. 2017.

NTALI, G.; KASSI, E.; ALEVIZAKI, M. Endocrine sequelae of immune checkpoint inhibitors. Hormones. Athens, Greece, v. 16, n. 4, p. 341-350, oct. 2017. 
O'MALLEY, G. et al. Rapid evolution of thyroid dysfunction in patients treated with nivolumab. Endocrine Practice, Jacksonville, v. 23, n. 10, p. 1223-1231, 2017.

ORLOV, S. et al. Induction of painless thyroiditis in patients receiving programmed death 1 receptor immunotherapy for metastatic malignancies. J Clin Endocrinol Metab., Hobokenm, v. 100, n. 5, p. 1738-1741, may. 2015.

PATEL, N.S. et al. Incidence of Thyroid Function Test Abnormalities in Patients Receiving Immune-Checkpoint Inhibitors for Cancer Treatment. The Oncologist, Durham, v. 23, n. 10, p. 1236-1241, 2018.

ROSS, D. S. et al. 2016 American Thyroid Association guidelines for diagnosis and management of hyperthyroidism and other causes of thyrotoxicosis. Thyroid, Falls Church, v. 26, n. 10, p. 1343-1421, 2016.

RYDER, M. et al. Endocrine-related adverse events following ipilimumab in patients with advanced melanoma: a comprehensive retrospective review from a single institution. Endocr Relat Cancer. Bristol, v. 21, n. 2, p. 371-81, mar. 2014.

SOUSA, R. B. et al. Incidence of endocrine dysfunction following the use of different immune checkpoint inhibitor regimens: a systematic review and meta-analysis. JAMA oncology, Chicago, v. 4, n. 2, p.173-182, 2018.

SOUZA, A. W. S. de. et al. Sistema Imunitário - Parte III. O delicado equilíbrio do sistema imunológico entre os pólos de tolerância e autoimunidade. Rev Bras Reumatol, São Paulo, v. 50, n. 6, p. 665-694, nov. 2010.

SPAIN, L. et al. Management of toxicities of immune checkpoint inhibitors. Cancer treatment reviews, United Kingdom, v. 44, p. 51-60, 2016.

TANAKA, R. et al. Nivolumab-induced thyroid dysfunction. Jpn J Clin Oncol., Oxford, v. 46, n. 6, p. 575-579, jun. 2016.

TORINO, F.; CORSELLO, S. M.; SALVATORI, R. Endocrinological side-effects of immune checkpoint inhibitors. Curr Opin Oncol. London, v. 28, n. 4, p. 278-287, jul. 2016.

TRAINER, H.; HULSE, P.; HIGHAM, C.E.; et al. Hyponatraemia secondary to nivolumabinduced primary adrenal failure. Endocrinol Diabetes Metab Case Rep, Bristol, v. $2016 ; 2016$

WAINSTEIN, A. J. et al. Diretrizes brasileiras de manejo de toxicidades imunomediadas associadas ao uso de bloqueadores de correceptores imunes. Brazilian Journal Oncology, São Paulo, v. 13, n. 4, p. 1-15, 2017.

WANG, D. Y. et al. Fatal toxic effects associated with immune checkpoint inhibitors: a systematic review and meta-analysis. JAMA oncology, Chicago, v. 4, n. 12, p. 1721-1728, 2018.

WEBER, J. S. et al. Management of Immune-Related Adverse Events and Kinetics of Response With Ipilimumab. J Clin Oncol, Oxford, v. 30, n. 21, p. 2691-2697, 2012 


\section{ANEXO A - APROVAÇÃO DO COMITÊ DE ÉTICA EM PESQUISA}

\section{CENTRO UNIVERSITÁRIO DE BRASÍLIA - UNICEUB}

\section{PARECER CONSUBSTANCIADO DO CEP}

\section{DADOS DO PROJETO DE PESQUISA}

Título da Pesquisa: Análise de Disfunções Tireoidianas Induzidas pelo Tratamento Oncológico Imunoterápico.

Pesquisador: GABRIELA RESENDE VIEIRA DE SOUSA

Área Temática:

Versão: 3

CAAE: 74772317.5 .0000 .0023

Instituição Proponente: Centro Universitário de Brasília - UNICEUB

Patrocinador Principal: Financiamento Próprio

DADOS DO PARECER

Número do Parecer: 2.424.297

Situação do Parecer:

Aprovado

Necessita Apreciação da CONEP:

Não

BRASILIA, 08 de Dezembro de 2017

Assinado por:

Marilia de Queiroz Dias Jacome

(Coordenador) 